\title{
Core Structure and Non-Abelian Reconnection of Defects in a Biaxial Nematic Spin-2 Bose-Einstein Condensate
}

\author{
Magnus O. Borgh* and Janne Ruostekoski \\ Mathematical Sciences, University of Southampton, SO17 1BJ, Southampton, United Kingdom
}

(Dated: January 11, 2017)

\begin{abstract}
We calculate the energetic structure of defect cores and propose controlled methods to imprint a nontrivially entangled vortex pair that undergoes non-Abelian vortex reconnection in a biaxial nematic spin-2 condensate. For a singular vortex, we find three superfluid cores in addition to depletion of the condensate density. These exhibit order parameter symmetries that are different from the discrete symmetry of the biaxial nematic phase, forming an interface between the defect and the bulk superfluid. We provide a detailed analysis of phase mixing in the resulting vortex cores and find an instability dependent upon the orientation of the order parameter. We further show that the spin-2 condensate is a promising system for observing spontaneous deformation of a point defect into an "Alice ring" that has so far avoided experimental detection.
\end{abstract}

Topological defects and textures are ubiquitous across physical systems that seemingly have little in common [1], from liquid crystals [2] and superfluids [3] to cosmic strings [4]. They arise generically from symmetries of a ground state that is described by an order parameter [5] - a function parametrizing the set of physically distinguishable, energetically degenerate states. In the simple example of a scalar superfluid, the order parameter is the phase of the macroscopic wave function. More generally it may be a vector or tensor that is symmetric under particular transformations. In a uniaxial nematic (UN), the order parameter is cylindrically symmetric around a locally defined axis. It also exhibits a twofold discrete symmetry under reversal of the cylinder axis, which leads to half-quantum vortices (HQVs) in atomic spinor BoseEinstein condensates (BECs) [6 9] and superfluid liquid ${ }^{3} \mathrm{He}$ [10], and to $\pi$-disclinations in liquid crystals [1, 2]. In a biaxial nematic (BN), also the cylindrical symmetry is broken into the fully discrete symmetry of a rectangular brick, with dramatic consequences: the $\mathrm{BN}$ is the simplest order parameter that supports non-Abelian vortices that do not commute [5, 11]. As a result, colliding non-Abelian vortices cannot reconnect without leaving traces of the process, but must instead form a connecting rung vortex. Noncommuting defects appear as cosmic strings in theories of the early Universe [4], and have been predicted in BN liquid crystals [11].

Despite long-standing experimental efforts, BN phases in liquid crystals have experimentally proved more elusive than originally anticipated [12]. In atomic systems, the topological classification and dynamics of non-Abelian vortices have theoretically been studied in the cyclic phase of spin-2 [13 16] and in spin-3 BECs [17], though it remains uncertain whether any alkali-metal atoms exhibit the corresponding ground states. Consequently, any physical system where non-Abelian defects may be reliably studied is still lacking.

Spin-2 BECs exhibit - in addition to the ferromagnetic (FM) and cyclic phases - both UN and BN phases 1820], which, however, are degenerate at the mean-field level. Beyond mean-field theory, the degeneracy may be lifted by quantum fluctuations through interactiondependent "order-by-disorder" processes [18, 19].

Here, we instead break the degeneracy already at the mean-field level with a Zeeman shift and compute the energetically (meta)stable defect structures as well as propose a scheme for experimentally preparing vortices exhibiting non-Abelian reconnection. This shows how BN spin-2 BECs can provide an experimentally simple path to study structure and dynamics of non-Abelian defects. For an energetically stable singular vortex in a rotating system, we fully characterize the appearance of three superfluid core structures, in addition to the empty core. As well as one FM and one cyclic order-parameter core, a third superfluid core appears that exhibits a twofold symmetry, with cyclic and BN phases appearing offset inside the core. We provide a parametrization of the vortex wave function that captures the symmetry breaking and mixing of phases in the core. The structure of the superfluid cores results from a combination of energetics and topology as an interface forms between the BN phase of the bulk superfluid and the different symmetry of the core order parameter. Considering the cyclic core, we find that the discrete point group symmetry not only manifests itself in an anisotropic core shape, but also leads to an orientation-dependent instability, when the vortex line is sufficiently tilted with respect to the orientation of the order parameter.

The study of non-Abelian vortex dynamics requires preparation of a vortex pair with noncommuting topological charges. The order-parameter transformations corresponding to each vortex combine nontrivially, leading to a complicated wave function that is not amenable to conventional imprinting techniques. We propose a two-step protocol to imprint the vortex pair, based on rotating the effective magnetic field between the preparation of each vortex. This allows the imprinting to make use of simple representations of each vortex in a changing spinor basis. We numerically demonstrate the non-Abelian vortex reconnection. The proposed method could be general- 
ized to controlled creation of systems of noncommuting vortices in atomic superfluids.

We also show how a BN spin-2 BEC is a promising system to observe spontaneous deformation of a point defect into a HQV ring, called an "Alice ring" 21], whose detection has been beyond experimental resolution in spin-1 BECs despite considerable efforts 22, 23]. Alice rings are originally known from high-energy physics [24, 25] and represent a direct consequence of the topological "hairyball theorem."

Experimental interest in topological defects and textures in spinor BECs is currently accelerating. Recent efforts in spin-1 BECs have led to the in situ observation of a singly quantized vortex splitting into a pair of HQVs 9], confirming theoretical prediction 8], and to controlled preparation of coreless-vortex textures 26 28], the analogs of Dirac [22] and 't Hooft-Polyakov [29] monopoles, and particlelike solitons [30]. Our results for spin-2 reveal a defect-structure phenomenology considerably richer than that in the spin-1 BECs 8, 3134]. Preparation of spin-2 BECs has been achieved using ${ }^{87} \mathrm{Rb}$ [27, 35 37], which, like ${ }^{23} \mathrm{Na}$, is predicted to exhibit the nematic phases [20, 38]. The BN phase can then be realized by controlling the quadratic level shift, e.g., by microwave dressing [39] or laser fields [40]. Both ${ }^{87} \mathrm{Rb}$ and ${ }^{23} \mathrm{Na}$ are commonly used in spinor-BEC experiments, making the BN phase the most likely candidate for realization of a ground-state manifold supporting nonAbelian defects.

We write the mean-field-theoretical spin-2 condensate wave function in terms of the density $n(\mathbf{r})$ and a normalized spinor $\zeta(\mathbf{r})$ as $\Psi(\mathbf{r})=\sqrt{n(\mathbf{r})}\left(\zeta_{+2}(\mathbf{r}), \zeta_{+1}(\mathbf{r}), \zeta_{0}(\mathbf{r}), \zeta_{-1}(\mathbf{r}), \zeta_{-2}(\mathbf{r})\right)^{T}$. The Hamiltonian density then reads [20]

$$
\mathcal{H}=h_{0}+\frac{c_{0}}{2} n^{2}+\frac{c_{2}}{2} n^{2}|\langle\hat{\mathbf{F}}\rangle|^{2}+\frac{c_{4}}{2} n^{2}\left|A_{20}\right|^{2}+\mathcal{H}_{Z}(\langle\hat{\mathbf{F}}\rangle),
$$

where $h_{0}=\left(\hbar^{2} / 2 m\right)|\nabla \Psi|^{2}+\left(m \omega^{2} r^{2} / 2\right) n$, for atomic mass $m$ and an isotropic trap with frequency $\omega$. The spin operator $\hat{\mathbf{F}}$ is given by a vector of spin-2 Pauli matrices. In addition to the spin-independent and $|\langle\hat{\mathbf{F}}\rangle|^{2}$-dependent interaction energies, a third interaction term arises proportional to $\left|A_{20}(\mathbf{r})\right|^{2}=\frac{1}{5}\left|2 \zeta_{+2} \zeta_{-2}-2 \zeta_{+1} \zeta_{-1}+\zeta_{0}^{2}\right|^{2}$, where $A_{20}$ is the amplitude of spin-singlet pair formation [41]. The interaction strengths are given by the $s$-wave scattering lengths $a_{f}$ in the spin- $f$ channels of colliding spin-2 atoms as $c_{0}=4 \pi \hbar^{2}\left(3 a_{4}+4 a_{2}\right) / 7 m, c_{2}=$ $4 \pi \hbar^{2}\left(a_{4}-a_{2}\right) / 7 m$, and $c_{4}=4 \pi \hbar^{2}\left(3 a_{4}-10 a_{2}+7 a_{0}\right) / 7 m$. Finally, $\mathcal{H}_{Z}(\langle\hat{\mathbf{F}}\rangle)=p n\left\langle\hat{F}_{z}\right\rangle+q n\left\langle\hat{F}_{z}^{2}\right\rangle$ represents linear and quadratic Zeeman shifts of strengths $p$ and $q$, respectively.

When the Zeeman shifts are small, the spin-2 BEC exhibits three distinct, interaction-dependent groundstate phases [20, 41, 42]. These may be characterized in terms of $|\langle\hat{\mathbf{F}}\rangle|$ and $\left|A_{20}\right|$ : a spin-2 FM phase with $|\langle\hat{\mathbf{F}}\rangle|=2$ and $\left|A_{20}\right|=0$, a cyclic phase with
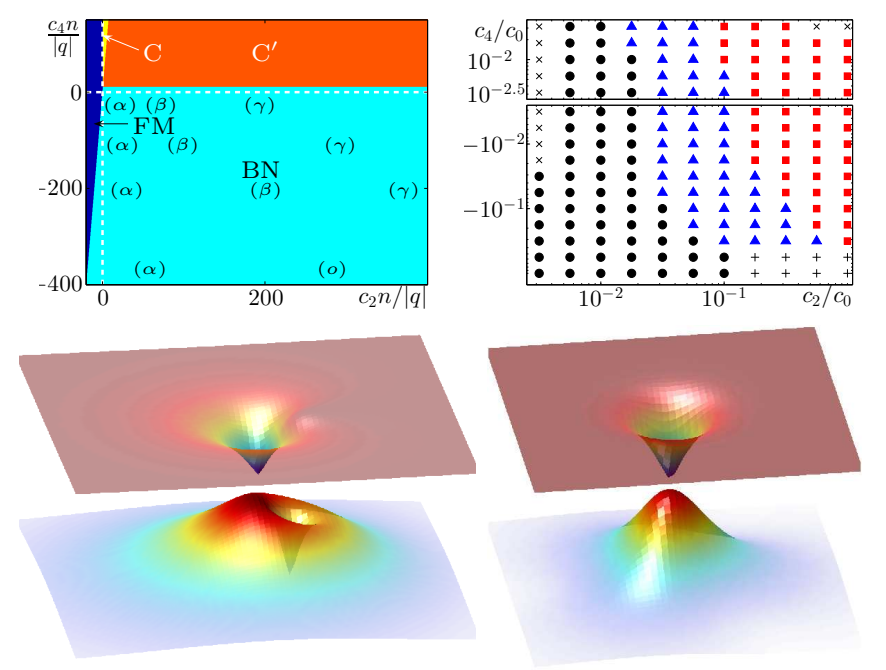

FIG. 1. Top: Ground-state phase diagram for $q<0$ with cores of $(1 / 2, \sigma)$ vortex indicated (left) and detailed core phase diagram (right): $[(o)$, plus $]$ empty, $[(\alpha)$, dot $] \mathrm{FM},[(\beta)$, triangle] cyclic, $[(\gamma)$, square] coexistence of and cyclic and phasemixing cores; (cross) single vortex unstable. Bottom: $\left|A_{20}\right|$ (top surface) and $\left|A_{30}\right|$ (bottom surface) in the phase-mixing (left) and cyclic (right) cores.

$|\langle\hat{\mathbf{F}}\rangle|=\left|A_{20}\right|=0$, and the polar phase with $|\langle\hat{\mathbf{F}}\rangle|=0$ and $\left|A_{20}\right|^{2}=1 / 5$. A polar spinor can be written as both $\zeta^{\mathrm{UN}}=(0,0,1,0,0)^{T}$ and $\zeta^{\mathrm{BN}}=(1 / \sqrt{2}, 0,0,0,1 / \sqrt{2})^{T}$, representing the $\mathrm{UN}$ and $\mathrm{BN}$ phases, respectively. These are energetically degenerate for $p=q=0[18,19]$. They may be distinguished by the amplitude of spin-singlet trio formation [41] $A_{30}=(3 \sqrt{6} / 2)\left(\zeta_{+1}^{2} \zeta_{-2}+\zeta_{-1}^{2} \zeta_{+2}\right)+$ $\zeta_{0}\left(\zeta_{0}^{2}-3 \zeta_{+1} \zeta_{-1}-6 \zeta_{+2} \zeta_{-2}\right)$, taking values $\left|A_{30}\right|^{2}=0,1$, and 2 in BN, UN, and cyclic phases, respectively.

With a nonzero quadratic Zeeman shift along the $z$ direction, $\zeta^{\mathrm{BN}}$ acquires an energy $\mathcal{H}_{Z}=4 q n$, while the energy of $\zeta^{\mathrm{UN}}$ is unchanged, breaking the degeneracy and energetically favoring the BN phase whenever $q<0$. By controlling the quadratic level shift [39, 40], the $\mathrm{BN}$ phase could be realized by experimentally simple means. The ground-state phase diagram is shown in Fig. 1. The BN phase is the ground state for $c_{2}>c_{4} / 20$ and $c_{4}<10|q| / n$, bordering the FM phase and two phases that continuously become cyclic for $|q| \rightarrow 0^{-}[20,43]$.

Axisymmetric vortices in the polar interaction regime were studied in Ref. [46]. With the energetic degeneracy between UN and BN phases lifted, we now separately consider their defects. While the UN phase shares common features with the spin-1 polar phase, the spin-2 BN phase represents a more drastic departure from the familiar defect families.

Mathematically, topologically distinguishable vortices correspond to the conjugacy classes of the first homotopy group $\pi_{1}(\mathcal{M})$ of the order-parameter space $\mathcal{M}$, and are determined by the group of transformations that keep the order parameter invariant [5]. Any BN spinor can 
be found by applying a condensate phase $\tau \in \mathrm{U}(1)$ and a spin rotation $\mathcal{R} \in \mathrm{SO}(3)$ to $\zeta^{\mathrm{BN}}$. The fourfold discrete symmetry means that $\zeta^{\mathrm{BN}}$ is left invariant by transformations in the eight-element group $\tilde{\mathrm{D}}_{4}$ that combines the dihedral-4 subgroup of $\mathrm{SO}(3)$ with elements of $\mathrm{U}(1)$ and factorizes $\mathrm{U}(1) \times \mathrm{SO}(3)$ to form $\mathcal{M}$. By lifting $\mathrm{SO}(3)$ to $\mathrm{SU}(2)$ to form a simply connected covering group, the conjugacy classes of $\pi_{1}(\mathcal{M})$ are obtained using standard techniques [43]: $\{(n, \mathbf{1})\},\{(n,-\mathbf{1})\}$, $\left\{\left(n, \pm i \sigma_{x}\right),\left(n, \pm i \sigma_{y}\right),\left(n, \pm i \sigma_{z}\right)\right\}, \quad\{(n+1 / 2, \sigma),(n+$ $\left.\left.1 / 2,-i \sigma_{z} \sigma\right)\right\},\left\{(n+1 / 2,-\sigma),\left(n+1 / 2, i \sigma_{z} \sigma\right)\right\}$, and $\{(n+$ $\left.\left.1 / 2, \pm i \sigma_{x} \sigma\right),\left(n+1 / 2, \pm i \sigma_{y} \sigma\right)\right\}$, where the Pauli matrices $\sigma_{x, y, z}$ and $\sigma \equiv\left(\mathbf{1}+i \sigma_{z}\right) / \sqrt{2}$ represent the $\mathrm{SU}(2)$ part of the $\pi_{1}(\mathcal{M})$ elements, and $n$ in the $\mathrm{U}(1)$ part is an integer. For $n=0$, we then identify: $(i)$ the vortex-free state, (ii) integer spin vortex, (iii) spin HQV, (iv) HQV with $\pi / 2$ spin rotation, $(v) \mathrm{HQV}$ with $3 \pi / 2$ spin rotation, and (vi) HQV with $\pi$ spin rotation.

We now show that a rich phenomenology of core states, resulting from the proliferation of spin-2 phases, appears in the $\mathrm{BN}$ defects. We consider the $(1 / 2, \sigma)$ vortex [case $(i v)]$, which is the simplest BN vortex that carries a mass circulation, and fully characterize its interactiondependent core structures. A prototype wave function for the vortex can be constructed by applying the corresponding condensate-phase winding and spin rotation to $\zeta^{\mathrm{BN}}: \zeta^{1 / 2, \sigma}=e^{i \phi / 2} e^{-i F_{z} \phi / 4} \zeta^{\mathrm{BN}}=\left(1,0,0,0, e^{i \phi}\right)^{T} / \sqrt{2}$, where $\phi$ is the azimuthal coordinate. The energetically stable core structure is determined by numerically minimizing the energy in the frame rotating with frequency $\Omega$, corresponding to rotation of the system, e.g., from a stirring potential. This is done by propagating the Gross-Pitaevskii equations [47] derived from Eq. (10) in imaginary time, taking $\zeta^{1 / 2, \sigma}$ as the initial state, including a global spin rotation to ensure nonzero population in all spinor components. We take the system-rotation axis to coincide with the effective magnetic field, but will later relax this assumption. We choose $N c_{0}=5000 \hbar \omega \ell^{3}$ (where $\ell=\sqrt{\hbar / m \omega}$ ), keep $q<0$ fixed, and vary $c_{2}$ and $c_{4}$. For ${ }^{87} \mathrm{Rb}$, measurements for $c_{0}$ [48], $c_{2}$ and $c_{4}$ 38] predict a polar ground state with $c_{2} / c_{0} \simeq 0.0103$ and $c_{4} / c_{0} \simeq-0.0055$. Measurement uncertainties, however, cross over into the cyclic regime. Also ${ }^{23} \mathrm{Na}$ is predicted to exhibit polar interactions [20].

We find four different energetically stable core structures for the $(1 / 2, \sigma)$ vortex, which depend on the interaction parameters $c_{0,2,4}$ as indicated in Fig. 1 When the interaction strengths are comparable in magnitude, the superfluid density is depleted on the vortex line singularity [the (o) core]. However, usually $\left|c_{2,4}\right|<c_{0}$ and the core may remain superfluid. This can be understood from the density, spin, and singlet healing lengths $-\xi_{n}=\hbar / \sqrt{2 m\left|c_{0}\right| n}, \xi_{F}=\hbar / \sqrt{2 m\left|c_{2}\right| n}$, and $\xi_{A}=\hbar / \sqrt{2 m\left|c_{4}\right| n}$ - which determine the size of defect cores that take the wave function out of the ground state of a uniform superfluid. By expanding to the largest heal- ing length, the core can lower its gradient energy. When $c_{2}$ is small, such that $\xi_{A} \lesssim \xi_{F}$, the vortex develops a FM core $\left[(\alpha)\right.$ in Fig. 1 as energy relaxes. As $c_{2}$ increases, maintaining a FM core becomes increasingly costly, and it eventually gives way to a cyclic core $(\beta)$ as $\xi_{A} \gtrsim \xi_{F}$. However, for sufficiently large $c_{2}$, a third superfluid core $(\gamma)$ approaching the UN phase on the singular line becomes approximately energetically degenerate, and coexists, with the $(\beta)$ core. The relaxed structure is sensitive to the initial state.

The measured [38, 48] interaction strengths for ${ }^{87} \mathrm{Rb}$ predict an $(\alpha)$ core, close to the $(\alpha)-(\beta)$ boundary. For ${ }^{23} \mathrm{Na}$, the interactions [20] similarly predict a $(\beta)$ core close to the transition to bistability with the $(\gamma)$ core. The $(\beta)$ and $(\gamma)$ cores are especially intriguing, exhibiting a complex mixing of phases that breaks the axial symmetry (Fig. 1). The cyclic $(\beta)$ core exhibits a triangular cross section shown in Fig. 1. The deformation arises due to the mismatch between the rectangular-brick BN symmetry and the tetrahedral symmetry of the cyclic phase [49]. In Fig. 1 the system-rotation axis is taken to coincide with the direction of the effective field corresponding to the Zeeman shift, which fixes the orientation of the order parameter. Tilting the vortex line relative to the order parameter, we find an orientation-dependent instability. As the angle approaches $\pi / 2$, the smooth connection of the $\mathrm{BN}$ and cyclic point-group symmetries is no longer possible, and the $(1 / 2, \sigma)$ vortex becomes unstable, giving way to a singly quantized vortex [43].

In the $(\gamma)$ core, the condensate approaches the UN phase on the line singularity, but exhibits a nonzero $|\langle\hat{\mathbf{F}}\rangle|$ as a result of the Zeeman energy. The core exhibits twofold symmetry, with cyclic and BN phases appearing offset on either side of the singular line as indicated by $\left|A_{30}\right|$ and $\left.\left|A_{20}\right|\right)$ in Fig. 1 (bottom left). This complex phase mixing is captured by the parametrization $\zeta^{(\gamma)}=$ $\left(\sqrt{1-g_{0}^{2}-g_{-2}^{2}}, 0, g_{0}(\rho), 0, g_{-2}(\rho) e^{i \phi}\right)^{T}$, with only three nonempty spinor components. The functions $g_{0}$ and $g_{-2}$ satisfy $g_{0}(\rho \rightarrow \infty)=0, g_{-2}(\rho \rightarrow \infty)=1 / \sqrt{2}$, and $g_{-2}(\rho \rightarrow 0)=0$. Then $\left|A_{30}\right|^{2}=-12 \cos (\phi) f(\rho)+h(\rho)$, where $f$ and $h$ are functions of $g_{0,-2}$, and the cosine term explains the local extrema. The value of $g_{0}$ on the vortex line is determined by energy minimization.

A striking consequence of the discrete BN symmetry is that the elements of $\pi_{1}(\mathcal{M})$, representing topological charges of line defects, do not all commute. Colliding vortices with commuting charges may pass through each other or reconnect without leaving traces of the process. For noncommuting vortices, these processes are forbidden by topology [5, 11]. They must instead reconnect by forming a rung vortex connecting the resulting defects. Non-Abelian defects have been proposed in BN liquid crystals 11 and theoretically classified in cyclic spin-2 BECs 13 16, 50 and in certain spin-3 phases 17].

We propose a controlled method of preparing a non- 


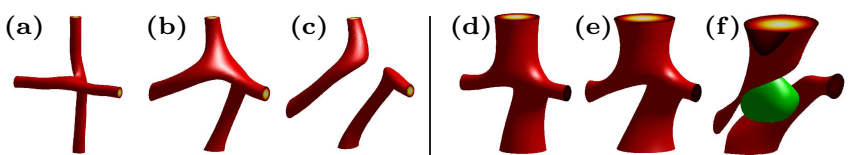

FIG. 2. Reconnection of $(\mathrm{a}-\mathrm{c})$ two $(1 / 2, \sigma)$ vortices, and $(\mathrm{d}-$ f) non-Abelian $(1 / 2, \sigma)$ and $\left(1 / 2, i \sigma_{x} \sigma\right)$ vortices. Red $|\langle\hat{\mathbf{F}}\rangle|$ isosurfaces indicate FM vortex cores. Green $\left|A_{30}\right|$ isosurface indicates the appearance of a rung vortex with uniaxial nematic core. Here $N c_{0}=1000 \hbar \omega \ell^{3}, c_{0} / c_{2}=100, c_{0} / c_{4}=-10$, and $q=-0.05 \hbar \omega$.

Abelian vortex pair and numerically demonstrate how it could realize a non-Abelian vortex reconnection in a $\mathrm{BN}$ spin-2 BEC. A nonoverlapping perpendicular vortex pair could be imprinted by a two-photon transition via an intermediate atomic level, with the singular phase profile of the driving electromagnetic field of each transition representing one vortex [51]. In a non-Abelian pair, however, the vortices are, in general, entangled in a complex way, making it challenging to imprint them, as the wave function no longer can be expressed as a combination of simple quantized vortex lines in individual spinor components. This is because the transformations of the BN order parameter corresponding to the $\mathrm{SU}(2)$ charges of each vortex line combine nontrivially. We therefore propose a two-step protocol where the magnetic field is rotated between the imprinting of the two vortices, making use of simple expressions of the vortex lines in different spinor basis representations [43]. We consider a $\pi / 2$ rotation that allows simple representations for both $(1 / 2, \sigma)$ and $\left(1 / 2, i \sigma_{x} \sigma\right)$ vortices [corresponding to the different HQV classes $(i v)$ and $(v i)]$ that do not commute. Moreover, imprinting the orientation of the vortex lines always along the $z$ axis of the changing spinor basis prepares the vortex lines perpendicular to each other, providing an ideal starting point for reconnection dynamics. The method can utilize the existing techniques of phase imprinting in spinor BECs by Raman transitions [52], and can also be applied, e.g., in the cyclic phase.

The corresponding reconnection dynamics is simulated by propagating the Gross-Pitaevskii equations, including a weak damping [43]. Figure 2] shows the reconnection and emergence of a rung vortex with UN core, characteristic of non-Abelian dynamics. For comparison we also show the reconnection of two $(1 / 2, \sigma)$ vortices, which trivially commute, and reconnect without forming a rung.

A distinguishing feature of UNs is the existence of topologically stable point defects [1, 5, 21, 53], which have very recently been observed in spinor BEC experiments [29]. Such a defect corresponds to a radial hedgehog texture of the nematic axis. In the spin-1 BEC, the point defect is predicted to relax into a $\mathrm{HQV}$ ring - an Alice ring - as a consequence of the hairy-ball theorem when the defect develops a superfluid core [21]. Alice rings are known in special electrodynamics models of

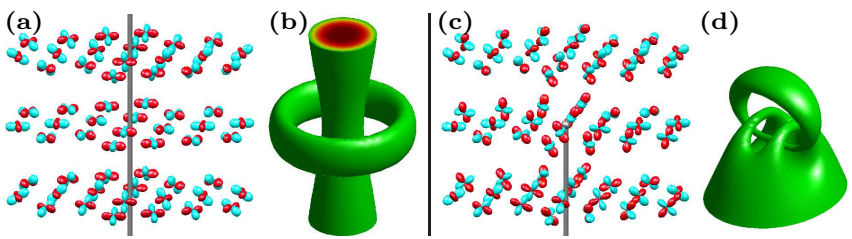

FIG. 3. (a), (c): BN order parameter exhibiting a radial hedgehog texture [cyan (light gray) lobes align with $\hat{\mathbf{r}}$ ] associated with a singular spin vortex indicated by a vertical line. (b), (d): Isosurface of $\left|A_{30}\right|$ showing the corresponding UN cores of spin vortices resulting from energy relaxation. In (c), a more elaborate construction allows the vortex to terminate at the point defect [43].

high-energy physics [24, 25]. Despite considerable experimental efforts, the vortex ring has not been observed in the spin-1 BEC [22], as on experimental time scales the size of the ring has remained too small to be detected [23].

In the $\mathrm{BN}$ condensate, the discrete order-parameter symmetry prohibits the formation of an isolated topologically stable point defect [1, 5]: any such a defect would be attached to a singular line. This is similar to the Dirac magnetic monopole, whose analog in the FM spin1 BEC [23, 54] was recently prepared in experiment [22].

The simplest way to construct a point defect associated with a vortex line in the $\mathrm{BN}$ spin- 2 BEC is to align one of the principal axes of the order parameter with $\hat{\mathbf{r}}$, as illustrated in Fig. 31(a) using a spherical-harmonics representation [43]. This creates a singular spin-vortex line along the $z$ axis, which develops a UN core as energy relaxes [Fig. 3(b)]. Additionally, energy relaxation causes the point-defect texture in the $\mathrm{BN}$ order parameter to spontaneously deform into a vortex ring, encircling the line defect. We identify this as a spin HQV [class (iii)]. We simulate the time evolution of the point defect for a spin- ${ }^{87} \mathrm{Rb}$ BEC and find that it provides a promising system where the intriguing deformation of a point defect into an Alice ring could be experimentally observed. In spin-1 BEC point-defect experiments [22] the size of the ring was estimated to be $\sim 0.2 \mu \mathrm{m}[23]$. In the spin-2 BEC, we numerically find characteristically faster expansion resulting in an order of magnitude larger ring size than in spin- ${ }^{23} \mathrm{Na}$ or ${ }^{87} \mathrm{Rb}$ BEC over experimental time scales, which is already within an achievable measurement resolution [22].

In conclusion, we have characterized the coexisting superfluid core structures of singular defects in a BN spin-2 BEC, and proposed how vortices exhibiting non-Abelian reconnection dynamics may be prepared in experiment. The realization of $\mathrm{BN}$ defects in the atomic system opens up several intriguing possibilities: Nucleation dynamics of non-Abelian defects could be studied in the spinorBEC Kibble-Zurek mechanism [55] by cooling through the BEC transition, or ramping the Zeeman shift into the $\mathrm{BN}$ regime. The connection of defects across a coher- 
ent interface between phases with different broken symmetry, e.g., mimicking the physics of string-theoretical branes [56, 57], could be studied using techniques similar to those proposed for spin-1 BECs [58, 59]. This forms a particularly intricate problem when one phase supports non-Abelian defects. The BN spin-2 BEC can also provide an experimentally simple path to non-Abelian turbulence scenarios 16]. Preparation of a point defect could lead to the observation of spontaneous deformation of a spherically symmetric core to an Alice ring.

The data presented can be found in Ref. [60].

We acknowledge financial support from the EPSRC. The numerical results were obtained using the Iridis 4 high-performance computing facility at the University of Southampton. We thank T. J. Sluckin for discussions.

* Current address: Faculty of Science, University of East Anglia, Norwich, NR4 7TJ, United Kingdom

[1] L. M. Pismen, Vortices in Nonlinear Fields (Oxford University Press, Oxford, 1999).

[2] M. Kleman and O. D. Lavrentovich, Soft Matter Physics: An Introduction (Springer, New York, 2003).

[3] G. E. Volovik, The Universe in a Helium Droplet (Oxford University Press, Oxford, 2003).

[4] A. Vilenkin and E. P. S. Shellard, Cosmic Strings and Other Topological Defects (Cambridge University Press, Cambridge, England, 1994).

[5] N. D. Mermin, Rev. Mod. Phys. 51, 591 (1979).

[6] U. Leonhardt and G. Volovik, JETP Lett. 72, 46 (2000).

[7] F. Zhou, Int. J. Mod. Phys. B 17, 2643 (2003).

[8] J. Lovegrove, M. O. Borgh, and J. Ruostekoski, Phys. Rev. A 86, 013613 (2012)

[9] S. W. Seo, S. Kang, W. J. Kwon, and Y.-i. Shin, Phys. Rev. Lett. 115, 015301 (2015).

[10] D. Vollhardt and P. Wölfle, The Superfluid Phases of Helium 3 (Taylor \& Francis Ltd, London, 1990).

[11] V. Poenaru and G. Toulouse, J. Phys. (Paris) 38, 887 (1977).

[12] G. R. Luckhurst and T. J. Sluckin, Biaxial Nematic Liquid Crystals: Theory, Simulation and Experiment (Wiley, Chichester, 2015).

[13] G. W. Semenoff and F. Zhou, Phys. Rev. Lett. 98, 100401 (2007).

[14] J. A. M. Huhtamäki, T. P. Simula, M. Kobayashi, and K. Machida, Phys. Rev. A 80, 051601 (2009).

[15] M. Kobayashi, Y. Kawaguchi, M. Nitta, and M. Ueda, Phys. Rev. Lett. 103, 115301 (2009).

[16] T. Mawson, G. Ruben, and T. Simula, Phys. Rev. A 91, 063630 (2015).

[17] R. Barnett, A. Turner, and E. Demler, Phys. Rev. A 76, 013605 (2007).

[18] A. M. Turner, R. Barnett, E. Demler, and A. Vishwanath, Phys. Rev. Lett. 98, 190404 (2007).

[19] J. L. Song, G. W. Semenoff, and F. Zhou, Phys. Rev. Lett. 98, 160408 (2007).

[20] Y. Kawaguchi and M. Ueda, Phys. Rep. 520, 253 (2012)

[21] J. Ruostekoski and J. R. Anglin, Phys. Rev. Lett. 91, 190402 (2003).
[22] M. W. Ray, E. Ruokokoski, S. Kandel, M. Möttönen, and D. S. Hall, Nature 505, 657 (2014).

[23] K. Tiurev, E. Ruokokoski, H. Mäkelä, D. S. Hall, and M. Möttönen, Phys. Rev. A 93, 033638 (2016)

[24] A. S. Schwarz, Nucl. Phys. B 208, 141 (1982).

[25] M. Alford, K. Benson, S. Coleman, J. Marchrussel, and F. Wilczek, Nucl. Phys. B 349, 414 (1991).

[26] A. E. Leanhardt, Y. Shin, D. Kielpinski, D. E. Pritchard, and W. Ketterle, Phys. Rev. Lett. 90, 140403 (2003).

[27] L. S. Leslie, A. Hansen, K. C. Wright, B. M. Deutsch, and N. P. Bigelow, Phys. Rev. Lett. 103, 250401 (2009).

[28] J.-y. Choi, W. J. Kwon, and Y.-i. Shin, Phys. Rev. Lett. 108, 035301 (2012).

[29] M. W. Ray, E. Ruokokoski, K. Tiurev, M. Möttönen, and D. S. Hall, Science 348, 544 (2015).

[30] D. S. Hall, M. W. Ray, K. Tiurev, E. Ruokokoski, A. H. Gheorghe, and M. Möttönen, Nat. Phys. 12, 478 (2016).

[31] S.-K. Yip, Phys. Rev. Lett. 83, 4677 (1999).

[32] T. Mizushima, K. Machida, and T. Kita, Phys. Rev. A 66, 053610 (2002).

[33] A.-C. Ji, W. M. Liu, J. L. Song, and F. Zhou, Phys. Rev. Lett. 101, 010402 (2008).

[34] J. Lovegrove, M. O. Borgh, and J. Ruostekoski, Phys. Rev. A 93, 033633 (2016).

[35] H. Schmaljohann, M. Erhard, J. Kronjäger, M. Kottke, S. van Staa, L. Cacciapuoti, J. J. Arlt, K. Bongs, and K. Sengstock, Phys. Rev. Lett. 92, 040402 (2004).

[36] M.-S. Chang, C. D. Hamley, M. D. Barrett, J. A. Sauer, K. M. Fortier, W. Zhang, L. You, and M. S. Chapman, Phys. Rev. Lett. 92, 140403 (2004).

[37] T. Kuwamoto, K. Araki, T. Eno, and T. Hirano, Phys. Rev. A 69, 063604 (2004).

[38] A. Widera, F. Gerbier, S. Fölling, T. Gericke, O. Mandel, and I. Bloch, New J. Phys. 8, 152 (2006).

[39] F. Gerbier, A. Widera, S. Fölling, O. Mandel, and I. Bloch, Phys. Rev. A 73, 041602 (2006).

[40] L. Santos, M. Fattori, J. Stuhler, and T. Pfau, Phys. Rev. A 75, 053606 (2007).

[41] M. Ueda and M. Koashi, Phys. Rev. A 65, 063602 (2002).

[42] C. V. Ciobanu, S.-K. Yip, and T.-L. Ho, Phys. Rev. A 61, 033607 (2000).

[43] See Supplemental Material, which includes Refs. 44, 45], for details.

[44] H. Goldstein, Classical Mechanics, 2nd ed. (Addison Wesley, 1980).

[45] C. W. Gardiner, J. R. Anglin, and T. I. A. Fudge, J. Phys. B: At. Mol. Opt. Phys. 35, 1555 (2002)

[46] W. V. Pogosov, R. Kawate, T. Mizushima, and K. Machida, Phys. Rev. A 72, 063605 (2005).

[47] J. Javanainen and J. Ruostekoski, J. Phys. A Mat. Gen. 39, L179 (2006).

[48] N. N. Klausen, J. L. Bohn, and C. H. Greene, Phys. Rev. A 64, 053602 (2001).

[49] M. Kobayashi, Y. Kawaguchi, and M. Ueda, arXiv:0907.3716v2 (2011).

[50] H. Mäkelä, Y. Zhang, and K.-A. Suominen, J. Phys. A: Math. Gen. 36, 8555 (2003)

[51] J. Ruostekoski and Z. Dutton, Phys. Rev. A 72, 063626 (2005).

[52] A. Hansen, J. T. Schultz, and N. P. Bigelow, Optica 3, 355 (2016).

[53] H. T. C. Stoof, E. Vliegen, and U. Al Khawaja, Phys. Rev. Lett. 87, 120407 (2001).

[54] C. M. Savage and J. Ruostekoski, Phys. Rev. A 68, 
043604 (2003).

[55] L. E. Sadler, J. M. Higbie, S. R. Leslie, M. Vengalattore, and D. M. Stamper-Kurn, Nature 443, 312 (2006).

[56] D. I. Bradley, S. N. Fisher, A. M. Guenault, R. P. Haley, J. Kopu, H. Martin, G. R. Pickett, J. E. Roberts, and V. Tsepelin, Nat. Phys. 4, 46 (2008).

[57] S. Sarangi and S.-H. H. Tye, Phys. Lett. B 536, 185
(2002)

[58] M. O. Borgh and J. Ruostekoski, Phys. Rev. Lett. 109, 015302 (2012)

[59] M. O. Borgh, J. Lovegrove, and J. Ruostekoski, New J. Phys. 16, 053046 (2014).

[60] M. O. Borgh and DOI $10.5258 /$ SOTON/403649
J. Ruostekoski, 